\title{
Tetravalent RNA-lipoplex Cancer Vaccine
}

National Cancer Institute

\section{Source}

National Cancer Institute. Tetravalent RNA-lipoplex Cancer Vaccine. NCI Thesaurus.

Code C122396.

A RNA-lipoplex (RNA-LP)-based cancer vaccine containing four naked ribonucleic acid (RNA)-drug products (DPS) RBL001.1, RBL002.2, RBL003.1, and RBL004.1 encoding melanoma-associated antigens (MAAs) encapsulated in liposomes, with potential antineoplastic activity. Upon intravenous administration of the tetravalent RNA-lipoplex cancer vaccine, the liposomes protect the RNA from degradation in the bloodstream, travel to the spleen and are taken up by antigen-presenting cells (APCs); RNA is translocated to the cytoplasm and translated into the four tumor-associated proteins. The expressed proteins are processed and the human leukocyte antigen (HLA)-peptide complexes are presented to the immune system. This induces antigen-specific CD8+ and $\mathrm{CD} 4+\mathrm{T}$-cell responses against the four selected MAAs. 